\title{
FUNCTIONAL ROTATION NUMBERS FOR ONE-DIMENSIONAL MAPS
}

\author{
A. M. BLOKH
}

\begin{abstract}
We introduce functional rotation numbers and sets for one-dimensional maps (we call them $f$-rotation numbers and sets) and deduce some of their properties (density of f-rotation numbers of periodic points in the f-rotation set, conditions for the connectedness of the f-rotation set) from the spectral decomposition theorem for one-dimensional maps.
\end{abstract}

\section{INTRODUCTION}

The notion of the rotation number was introduced by Poincare [P] for circle homeomorphisms. Newhouse, Palis and Takens [NPT] extended it onto circle degree one maps, introduced the notion of rotation interval and proved some properties of rotation intervals; their work was continued by Ito in [I]. We summarize the properties of rotation intervals proven in [NPT], [I] in Theorem INPT but first let us introduce necessary notations and definitions. Let $f: S^{1} \rightarrow$ $S^{1}$ be a map of degree $1, \pi: \mathbb{R} \rightarrow S^{1}$ be the natural projection (i.e. the one which maps the interval $[0,1)$ onto the whole circle); let us fix a lifting $F$ of $f$. If $x \in S^{1}, X \in \pi^{-1} x$ then we denote the set of all limit points of the sequence $F^{n}(X) / n$ by $I_{F}(x)$; the notation is correct since for a degree one map $f$ the set $I_{F}(x)$ does not depend on the choice of $X \in \pi^{-1} x$. If $I_{F}(x)=\left\{\rho_{F}(x)\right\}$ is a one-point set then $\rho_{F}(x)$ is called the rotation number of $x$. Set $\bigcup_{x \in S^{1}} I_{F}(x) \equiv I_{F}$; the following theorem is proven in [NPT, I] (properties of circle maps without cycles may also be deduced from [AK]).

Theorem INPT ([I, NPT]). (1) $I_{F}$ is a closed interval.

(2) If $f$ has periodic points then the set of all rotation numbers of periodic points is dense in $I_{F}$; otherwise $f$ is monotonically semiconjugate to an irrational rotation by angle $2 \pi \alpha$ and $I_{F}=\{\alpha\}$.

Since liftings of the same degree one circle map $f$ differ by integers we may fix $F$ and use $f$ as a subscript from now on. The set $I_{f}$ is called the rotation set (interval) of $f$. Let us give another well-known interpretation for the rotation numbers and sets (see, e.g., [MZ2]). Namely, let the function $\phi_{f}: S^{1} \rightarrow \mathbb{R}$ be such that $\phi_{f}(z)=F(Z)-Z$ for some $Z \in \pi^{-1} z$; since $F\left(Z^{\prime}\right)-Z^{\prime}=F\left(Z^{\prime \prime}\right)-Z^{\prime \prime}$ whenever $\pi\left(Z^{\prime}\right)=\pi\left(Z^{\prime \prime}\right)$ the function $\phi_{f}$ is well defined and continuous. Then $I_{f}(z)$ is the set of limit points of the sequence

Received by the editors July 7, 1993.

1991 Mathematics Subject Classification. Primary 54H20, 58F03, 58F08.

Key words and phrases. Periodic points, rotation numbers, interval maps. 
$\frac{1}{n} \sum_{i=0}^{n-1} \phi\left(f^{i} z\right)$ and $I_{f}$ is the union of all such sets taken over all points of the circle. The natural question is to what extent Theorem INPT may be generalized for other functions and manifolds. In this paper we give necessary definitions and state sufficient conditions for the analog of Theorem INPT to be true in case of circle or interval maps and bounded measurable functions; we also outline some applications and possible extensions of our results onto maps of onedimensional branched manifolds. Our main tool is the "spectral decomposition theorem" for one-dimensional maps [B1-B3]. Also we would like to point out that related problems in symbolic dynamics are considered by $\mathrm{K}$. Ziemian [Z].

Let $X$ be a compact metric space, $\phi: X \rightarrow \mathbb{R}^{n}$ be a bounded measurable function, $P_{\phi}(\mu)=\int_{X} \phi d \mu$ for any measure $\mu$. Let also $f: X \rightarrow X$ be continuous. It is easy to see that the set $I_{f, \phi}(x)$ of limit points of the sequence $\frac{1}{n} \sum_{i=0}^{n-1} \phi\left(f^{i} x\right)$ is closed and connected. Let $V_{f}(x)$ be the set of all limit points of Cesàro averages of iterates of $\delta$-measure concentrated at $x \in X$; if $P_{\phi}$ is continuous on $V_{f}(x)$ then $I_{f, \phi}(x)=\phi\left(V_{f}(x)\right)$. It is well known that if $D_{\phi}$ is the set of all points of discontinuity of $\phi, \mu$ is a measure and $\mu\left(D_{\phi}\right)=0$ then $P_{\phi}$ is continuous at $\mu$. Let us say that a point $x$ is $\phi$-admissible if for any measure $\nu \in V_{f}(x)$ we have $\nu\left(D_{\phi}\right)=0$; if $x$ is $\phi$-admissible then $P_{\phi}$ is continuous on $V_{f}(x)$ and $I_{f, \phi}(x)=\phi\left(V_{f}(x)\right)$. Denote the set of all $\phi$-admissible points by $\operatorname{Ad}_{f, \phi}$. Note also that $V_{f}(x)$ is connected and closed [DGS].

We call $I_{f, \phi}(x)$ the $\phi$-rotation set of $x$; if $I_{f, \phi}(x)=\left\{\rho_{f, \phi}(x)\right\}$ is a onepoint set then $\rho_{f, \phi}(x)$ is called the $\phi$-rotation number of $x$. Also, if $\nu$ is an invariant measure then we call $\rho_{f, \phi}(x)=P_{\phi}(\nu)$ the $\phi$-rotation number of the measure $\nu$; in case of a periodic orbit we consider its rotation number even if $\phi$ is discontinuous at some of its points. Let $V_{f}=\bigcup_{x \in X} V_{f}(x), V_{f}(\phi)=$ $\bigcup_{x \in \operatorname{Ad}_{f, \phi}} V_{f}(x)$. We call the set $\phi\left(V_{f}(\phi)\right)=\bigcup_{x \in \operatorname{Ad}_{f, \phi}} I_{f, \phi}(x) \equiv I_{f}(\phi)$ (i.e. the set of all limit points of all sequences $\left.\left\{\frac{1}{n} \phi\left(f^{n} x\right)\right\}_{n=1}^{\infty}, x \in \operatorname{Ad}_{f, \phi}\right)$ the $\phi$-rotation set of $f$. Note that the union of all sets $I_{f, \phi}(x)$ may be bigger than $I_{f}(\phi)$ since $\phi$ may be discontinuous and so $\operatorname{Ad}_{f, \phi}$ may be very small. We call the above introduced rotation numbers and sets functional or f-rotation numbers and sets. Clearly if $\phi$ is continuous then $\operatorname{Ad}_{f, \phi}=X$; in particular if $f: S^{1} \rightarrow S^{1}$ is a degree one circle map then the function $\phi_{f}$ is continuous, $\operatorname{Ad}_{f, \phi}=S^{1}, I_{f}(z)=$ $I_{f, \phi_{f}}(z), \rho_{f}(z)=\rho_{f, \phi_{f}}(z)$ whenever $\rho_{f}(z)$ exists and $I_{f}\left(\phi_{f}\right)=I_{f}$.

Example 1. Let $f:[0,1] \rightarrow[0,1]$ be a mixing continuous map, $\phi(x)$ equals $1 / n$ if $x$ is periodic of period $n$ and 0 otherwise; then $I_{f}(\phi)=\{0\}$ and at the same time the union of all sets $I_{f, \phi}(x)$ is the union of all numbers $1 / k$ and 0 where $k$ is a period of a periodic point of $f$. Indeed by [B1, B3] the set of periodic points (which are exactly the points of discontinuity of $f$ ) is dense in $[0,1]$. The rest follows from the definitions. Note that even though periodic points do not belong to $\operatorname{Ad}_{f, \phi}$ the closure of the set of rotation numbers of periodic points contains $I_{f}(\phi)$ (since periods of periodic points are not bounded in this case).

Let $f: X \rightarrow X$ be an interval or the circle map. An interval $I$ is called periodic (of period $k$ ) or $k$-periodic if $J, \ldots, f^{k-1} J$ are pairwise disjoint and $f^{k} J \subset J$; the set $M=\bigcup_{i=0}^{k-1} f^{i} J \equiv$ orb $J$ is then called a cycle of intervals (we write also $\operatorname{per}(J)=\operatorname{per}(M)=k)$. Usually we assume that a periodic interval is closed and specify if it is not required. The map $f \mid M$ is called a nonstrictly 
periodic map; in particular if $X$ is an interval then $f: X \rightarrow X$ is nonstrictly periodic. The invariant probability measure concentrated on a periodic orbit (and thus equidistributed) is called a CO-measure [DGS]; denote the set of all such measures $C O(f)$. Theorem 1 is a simplified version of the result obtained in [B1, B3] for interval maps and in [B2] for graph maps.

Theorem 1 ([B1-B3]). Let $f: X \rightarrow X$ be a continuous circle map with $\operatorname{Per} f \neq \varnothing$ or nonstrictly periodic map, $\mu$ be an invariant probability measure. Then the following statements are equivalent:

(1) there exists $x$ such that $\mu(\omega(x))=1$;

(2) there exists a generic point for $\mu$ which can always be chosen from $\overline{\operatorname{Per} f}$;

(3) $\mu$ can be approximated arbitrary well by a CO-measure.

Corollary 1. $V_{f}=\overline{C O(f)}$ and so $V_{f}$ is closed.

The description of the dynamics for the circle maps without periodic points is well known [AK]; similar description holds for graph maps without periodic points [B2]. To state the result we need the following definition: a continuous map $\phi: X \rightarrow Y$ is called monotone if the preimage of any point is connected.

Theorem AK ([AK]). If $f: S^{1} \rightarrow S^{1}$ has no periodic points then $f$ is semiconjugate by a monotone map $\xi$ to an irrational rotation, there is an invariant perfect set $E \subset S^{1}$ with $\omega(x)=E(\forall x), \xi(E)=S^{1}, \xi \mid E$ is at most 2-to-1 and $f$ has a unique invariant measure $\mu_{f} ; \mu_{f}$ is mapped by $\xi$ onto the Lebesgue measure on $S^{1}$.

Let $\mathscr{P}$ be the family of all circle maps with periodic points united with the family of all interval maps, $\mathscr{R}$ be the family of all degree one circle maps without periodic points. This paper is devoted to studying f-rotation numbers and sets of maps from $\mathscr{P} \cup \mathscr{R}$. In fact the results about maps from $\mathscr{R}$ follow from Theorem AK, so we mainly study maps from $\mathscr{P}$. The following is an immediate corollary of Theorems 1 and AK.

Proposition 1. (1) Let $f \in \mathscr{P}$. Then the closure of the set of $\phi$-rotation numbers of periodic points of $f$ contains the rotation set $I_{f}(\phi)$.

(2) Let $f \in \mathscr{R}$. If $\mu_{f}\left(D_{\phi}\right)=0$ then $I_{f}(\phi)=\int \phi d \mu$ is a singleton, otherwise it is empty.

Propositions 2, 3 are also easy corollaries of Theorems 1 and AK.

Proposition 2. For any $\gamma \in I_{f}(\phi)$ there is a point $x \in \operatorname{Ad}_{f, \phi} \cap \overline{\operatorname{Per} f}$ such that $V_{f}(x)$ consists of a unique measure $\mu$ and $P_{\phi}(\mu)=\gamma$.

Proposition 3. If $\phi$ is continuous then $I_{f}(\phi)$ is closed.

We now describe a situation in which properties of $\phi$ imply that the set $I_{f}(\phi)$ is connected. First we need some definitions from [ALM, MN] related to socalled combinatorial dynamics. Let $X$ be the circle or the interval, $f: X \rightarrow X$ be a continuous map. Let $F: X^{\prime} \rightarrow X^{\prime}$ be a lifting of $f$ to the universal covering space $X^{\prime}$ of $X$ generated by the identity map if $X$ is the interval or by the natural projection if $X$ is the circle (so $F=f$ if $X$ is the interval and $F: \mathbb{R} \rightarrow \mathbb{R}$ if $X$ is the circle). Let $P, Q$ be two periodic orbits; say that $P$ represents the same pattern as $Q$ if for their liftings $P^{\prime}, Q^{\prime}$ there is a homeomorphism $h: X^{\prime} \rightarrow X^{\prime}$ which preserves the orientation and conjugates 
$F \mid P^{\prime}$ to $F \mid Q^{\prime}$; the class $\pi$ of all periodic orbits representing the same pattern as $P$ is called a pattern and so it is said that $P$ represents $\pi$ (we will also say that $P$ is of pattern $\pi$ and denote $\pi$ by $\operatorname{ptn}(P))$. Obviously patterns have well-defined periods. Moreover, let $P$ represent a pattern $\pi, Q$ represent a pattern $\theta$. If there is an increasing 2-to-1 semiconjugacy $h$ of $F \mid P^{\prime}$ to $F \mid Q^{\prime}$ then we say that $\pi$ is a 2-extension of $\theta$. If there are patterns $\pi_{1}, \ldots, \pi_{n}$ such that $\pi_{i+1}$ is a 2-extension of $\pi_{i}$ for $1 \leq i \leq n-1$ we say that $\pi_{n}$ is a simple extension of $\pi_{1}$; simple extensions of a fixed point pattern on the interval are called simple patterns [B12], periodic orbits which represent simple patterns are also called simple. Two patterns are called equivalent if they both are simple extensions of the same pattern; two periodic points are equivalent if they represent equivalent patterns. Theorem 2 which is our main result relies upon the decomposition theorem for one-dimensional maps [B1-B3].

Theorem 2. Let a function $\phi$ be such that for any two periodic orbits $P, Q$ we have $\rho_{f, \phi}(P)=\rho_{f, \phi}(Q)$ provided that $P$ is equivalent to $Q$. Then the following holds.

(1) Let $X$ be the circle and the set $D_{\phi}$ of discontinuities of $\phi$ belong to the set of all nonperiodic points of $f$. Then $I_{f}(\phi)$ is connected.

(2) Let $X$ be an interval and suppose there is an infinite sequence $A \subset \mathbb{Z}^{+}$ containing arbitrary big pairs of consecutive integers and such that set $D_{\phi}$ of discontinuities of $\phi$ belongs to the set of all nonperiodic points united with the set of all points from simple periodic orbits of periods $2^{n}, n \notin A$. Then $I_{f}(\phi)$ is connected.

Clearly Propositions 1, 3 and Theorem 2 imply Theorem INPT. To give another application of Theorems 1 and 2 we need the following definition. For an interval map $f:[0,1] \rightarrow[0,1]$ let the function $\chi$ be such that $\chi(x)=1$ if $f x<x$ and $\chi(x)=0$ if $f x>x$. Fix a number $n$, let $A^{\prime}$ be a subfamily of the family of all $n$-sequences of symbols 0,1 and define a function $\xi$ which depends on $A^{\prime}$ and has the properties from Theorem 2 as follows. For a point $x$ such that $f^{n-1} x$ is not fixed let $a_{i}=\chi\left(f^{i} x\right), 0 \leq i \leq n-1$; then $\xi(x)=1$ if $\left\{a_{i}\right\}_{i=0}^{n-1} \in A^{\prime}$ and 0 otherwise. To define $\xi$ at points $x$ such that $f^{n-1} x$ is fixed consider two sequences of $n$ symbols: $\bar{\eta}_{0}=\{0,1,0,1, \ldots\}$ and $\bar{\eta}_{1}=\{1,0,1,0, \ldots\}$ and let $\gamma$ be the number of those of them which belong to $A^{\prime}$ divided by 2 ; now let $\xi(x)=\gamma$ for any $x$ such that $f^{n-1} x$ is fixed.

It is easy to see that Theorem 2(2) is applicable to $\xi$. Indeed, let $x \in \operatorname{per} f$ be a nonfixed periodic point and $\bar{\alpha}(x)=\left\{a_{0}, a_{1}, \ldots\right\}$ be the infinite sequence of symbols 0 and 1 defined by $a_{k}=\chi\left(f^{k} x\right)$. Then $\xi$-rotation number of $x$ is completely determined by $\bar{\alpha}(x)$. On the other hand if $Q$ is equivalent to $P=$ orb $x$ then choosing an appropriate point $y \in Q$ we may assume that $\bar{\alpha}(y)=\bar{\alpha}(x)$ and thus $\xi$-rotation numbers of $P$ and $Q$ are the same. It remains to notice that for fixed points and their simple extensions the same property holds due to the definition of $\xi$. Since the only periodic points of discontinuity of $\xi$ are some of the fixed points of $f$ then Theorems 1 and 2 are applicable and we prove the following

Corollary 2. $I_{f}(\xi)$ is an interval, $\xi$-rotation numbers of periodic points are dense in $I_{f}(\xi)$.

If $n=1$ and the set $A^{\prime}$ of sequences of the length $n$ consists of the sequence 
$\{1\}$ (in this case $\xi=\chi$ outside the set of fixed points of $f$ and $1 / 2$ at fixed points, $\chi$-rotation numbers and sets are simply called rotation numbers and sets [B6]) then one can say more. Indeed, the following Corollary 3 is a weak version of the Main Theorem from [B6] (cf. [M2]); in this corollary speaking of the interval $(p / q, 1 / 2]$ we do not necessary assume that $p / q<1 / 2$.

Corollary 3 ([B6]). Let $f$ have a periodic point with the rotation number $p / q \neq$ $1 / 2$. Then for any positive integers $s, t$ such that $s / t \in(p / q, 1 / 2]$ there is an $f$-periodic point $y$ of period $t$ with the rotation number $s / t$.

Corollary 4 follows from Corollary 3, Proposition 1(1), and Theorem 2.

Corollary 4. If $p, q$ are such positive integers that $p / q \in$ int $I_{f}$ then there is an $f$-periodic point $x$ of rotation number $p / q$ and period $q$.

Acknowledgments. I would like to thank E. Coven and M. Misiurewicz for useful discussions of the results of this paper.

\section{Preliminaries}

In this section we describe for our purposes a short suitable version of the spectral decomposition for one-dimensional maps contained in [B1-B3]. More precisely, in [B1, B3] the full construction for interval maps with some corollaries is given. In [B2] this construction is extended for continuous graph maps, so the results of [B2] cover the case of continuous circle maps (in fact the circle case may be easily covered by slightly modified arguments from [B3]). We state results of [B1-B3] sometimes dividing statements into two parts, one concerning interval maps and taken directly from [B3] and the other one concerning circle maps and specializing results of [B2] for them. Since the description of circle maps without periodic points follows from Theorem AK we confine ourselves in this section to the family of maps $\mathscr{P}$. Let us briefly explain how limit sets are classified in [B1-B3]. Fix an infinite set $\omega(x)$ and consider the family $\mathscr{A}$ of all cycles of intervals orb $I$ such that $\omega(x) \subset$ orb $I$. There are two possibilities.

(1) Periods of sets orb $I \in \mathscr{A}$ are not bounded. Then there exist ordered cycles of intervals containing $\omega(x)$ with periods tending to infinity. This allows us to semiconjugate $f \mid \omega(x)$ to a transitive translation in a compact group and implies many properties of $f \mid \omega(x)$.

(2) Periods of sets orb $I \in \mathscr{A}$ are bounded. Then there exists a minimal cycle of intervals orb $J \in \mathscr{A}$. It is easy to see that all points $y \in \omega(x)$ have the following property: if $U$ is a neighborhood of $y$ in orb $J$ then orb $U=$ orb $J$ (otherwise orb $U$ generates a cycle of intervals orb $K$ such that $\omega(x) \subset$ orb $K \varsubsetneqq$ orb $J$ which is a contradiction). The idea is to consider all the points $z \in$ orb $J$ with this property. They form a set $B$ which turns out to be a maximal by inclusion limit set with some important properties.

Let us proceed more precisely. Let $I_{0} \supset I_{1} \supset \ldots$ be periodic intervals with periods $m_{0}, m_{1}, \ldots$. Obviously $m_{i+1}$ is a multiple of $m_{i}$ for all $i$. If $m_{i+1}>$ $m_{i}(i>0)$ then the intervals $\left\{I_{j}\right\}_{j=0}^{\infty}$ are said to be generating and any invariant closed set $S \subset Q=\bigcap_{j \geq 0}$ orb $I_{j}$ is called a solenoidal set; if $Q$ is nowhere dense then we call $Q$ a solenoid. We use the following notation: $\bigcap_{j \geq 0}$ orb $I_{j} \equiv$ $Q\left(\left\{I_{j}\right\}_{j=0}^{\infty}\right) \equiv Q, Q \cap \overline{\operatorname{Per} f} \equiv S_{p}(Q) \equiv S_{p}$. One can use a transitive translation 
in an Abelian zero-dimensional infinite group as a model for the map on a solenoidal set. Namely, let $D=\left\{n_{i}\right\}_{i=0}^{\infty}$ be a sequence of integers and $n_{i+1}>n_{i}$ be a multiple of $n_{i}$ for all $i$. Consider a group $H(D) \subset \mathbb{Z}_{n_{0}} \times \mathbb{Z}_{n_{1}} \times \ldots$, defined by $H(D) \equiv\left\{\left(r_{0}, r_{1}, \ldots\right): r_{i+1} \equiv r_{i}\left(\bmod m_{i}\right)(\forall i)\right\}$. The group operation is trivially defined; let $\tau$ be the (minimal) translation in $H(D)$ by the element $(1,1, \ldots)$. The following is a short version of the results of [B1-B3] describing properties of solenoidal sets.

Proposition 4 ([B1-B3]). Let $\left\{I_{j}\right\}_{j=0}^{\infty}$ be generating intervals of periods $\left\{m_{i}\right\}_{i=0}^{\infty}$ $=D, Q=\bigcap_{j \geq 0}$ orb $I_{j}$. Then there exists a continuous map $\phi: Q \rightarrow H(D)$ such that:

(1) $\phi$ semiconjugates $f \mid Q$ to $\tau$ (i.e. $\tau \circ \phi=\phi \circ f$ and $\phi$ is surjective);

(2) there exists a unique set $S \subset S_{p}$ such that $\omega(x)=S$ for any $x \in Q, f \mid S$ is minimal, the set $S_{p} \backslash S$ is at most countable set of isolated points and there is a unique measure $\nu_{Q}$ such that $\nu_{Q}(Q)=1$ (in fact supp $\left.\nu_{Q}=S\right)$;

(3) if $\omega(z) \cap Q \neq \varnothing$ then $S \subset \omega(z) \subset Q$ and $S$ is in fact equal to the set of all accumulation points of $\omega(z)$.

Theorem 2 implies the following

Corollary 5. For a point $x$ such that $\omega(x) \cap Q \neq \varnothing$ we have $\left.V_{f}(x)=\left\{\nu_{Q}\right)\right\}$.

Let us turn to another type of maximal infinite limit set. Let $\xi: K \rightarrow$ $K$ and $\xi^{\prime}: K^{\prime} \rightarrow K^{\prime}$ be nonstrictly periodic or circle maps, $K$ and $K^{\prime}$ be homeomorphic. Let $\phi: K \rightarrow K^{\prime}$ be a monotone semiconjugacy between $\xi$ and $\xi^{\prime}$ and $F \subset K$ be a $\xi$-invariant closed set such that $\phi(F)=K^{\prime}$, for any $x \in K^{\prime}$ we have int $\phi^{-1}(x) \cap F=\varnothing$ and so $\phi^{-1}(x) \cap F \subset \partial \phi^{-1}(x), 1 \leq$ $\operatorname{card}\left\{\phi^{-1}(x) \cap F\right\} \leq 2$. Then say that $\phi$ almost conjugates $\xi \mid F$ to $\xi^{\prime}$. Now, let $M$ be a cycle of intervals or a circle. Consider a set $\{x \in M$ : for any relative neighborhood $U$ of $x$ in $M$ we have orb $U=M\}$; clearly, this is a closed invariant set. It is called a basic set and denoted by $B(M, f)$ provided it is infinite; if $B(M, f)$ exists we say that $M$ generates a basic set. Proposition 5 describes properties of basic sets.

Proposition 5 ([B1-B3]). Let $B=B(M, f)$ be a basic set. Then $f \mid M$ is surjective and there exist a transitive map $g: M^{\prime} \rightarrow M^{\prime}, M^{\prime}$ homeomorphic to $M$, and a monotone map $\phi: M \rightarrow M^{\prime}$ such that $\phi$ almost conjugates $f \mid B$ to $g$. Furthermore, the following holds:

(a) $B \subset \overline{\operatorname{Per} f}$ is a perfect set;

(b) $f \mid B$ is transitive and if $\omega(z) \supset B$ then $\omega(z)=B$ (i.e. $B$ is a maximal limit set);

(c) $h(f \mid B)>0$;

(d) if $x \in B$ is not isolated in $B$ from a side $T$ then for any $T$-semineighborhood $U \subset M$ of $x$ we have $\overline{\operatorname{orb} U}=M$.

Proposition 5 shows that properties of maps on their basic sets are close to properties of transitive nonstrictly periodic or circle maps. We describe these properties in Proposition 6. To state it we need the following definition: an interval map $f:[a, b] \rightarrow[a, b]$ is called a flip if there is a point $c \in(a, b)$ such that $f[a, c]=[c, b], f[c, b]=[a, c]$.

Proposition 6 ([B1-B3]). Let $f: X \rightarrow X$ be a transitive nonstrictly periodic or circle map. Then either $f$ is mixing or there is an interval $I \subset X$ and a 
number $n>0$ such that $f^{n} I=I, \bigcup_{i=0}^{n-1} f^{i} I=X, f^{n} \mid I$ is mixing and distinct sets $f^{k} I, f^{j} I$ have at most finitely many common points. Moreover, if $X$ is a finite union of $k$ disjoint intervals then either $n=k$ or $n=2 k$ (in the latter case $f^{k}$ on any component of $X$ is a flip) and in any case $f$ has periodic points of all periods $2 n l, l>0$.

Let us introduce some notations. If $L$ is a cycle of intervals of period $r$ then the minimal period of periodic points from $L$ is $r$. All the periodic orbits of period $r$ from $L$ represent the same pattern denoted by $\operatorname{ptn}(L)$; we will sometimes say that $L$ represents this pattern. The following is an easy corollary of Proposition 5.

Corollary 6. (1) Let $B(M, f)$ be a basic set, $L \subset M$ be a cycle of intervals complementary to $B(M, f)$ in $M$. Then there is a periodic orbit $P^{\prime} \subset B(M, f) \cap L$ such that either $\operatorname{ptn}\left(P^{\prime}\right)=\operatorname{ptn}(L)$ or $\operatorname{ptn}\left(P^{\prime}\right)$ is a 2-extension of $\operatorname{ptn}(L)$.

(2) There is a periodic orbit $P^{\prime \prime} \subset B(M, f)$ such that either $\operatorname{ptn}\left(P^{\prime \prime}\right)=$ $\operatorname{ptn}(M)$ or $\operatorname{ptn}\left(P^{\prime \prime}\right)$ is a 2-extension of $\operatorname{ptn}(M)$.

Let $M$ be a cycle of intervals of period $k, B=B(M, f)$ be a basic set, $I=[a, b]$ be a component of $M$ and show that there is a periodic orbit $Q \in M$ of period $k$ such that if $\{x\}=Q \cap I$ then both $[a, x] \cap B(M, f)$ and $[x, b] \cap B(M, f)$ are infinite sets. Indeed, by Proposition $5 f^{k} \mid I$ is monotonically semiconjugate to a transitive interval map $g:[0,1] \rightarrow[0,1]$. Clearly there is at least one $g$-fixed point in $(0,1)$. Due to the monotonicity of the semiconjugacy its preimage is a $k$-periodic interval of $f$, thus it contains a $k$-periodic point of $f$ which has all the required properties; its orbit will be denoted by $Q(M)$. The following is an easy corollary of the definitions.

Corollary 7. If $N$ is a cycle of intervals such that $Q(M) \subset N \not \subset M$ then $N \supset M$. Proof. We use the notation introduced before Corollary 7. Then either $[a, x] \subset$ $N$ or $[x, b] \subset N$. We may assume that $[a, x] \subset N$; at the same time the choice of $x$ implies that there is at least one point $y$ in $B(M, f) \cap(a, x)$ which together with the definition of a basic set and the fact that $N$ is invariant imply that $M \subset N$ completing the proof.

Let us denote by $X_{f}$ the union of all periodic orbits which are maximal by inclusion among limit sets; together with solenoidal sets of type $S_{p}(Q)$ and basic sets they are the components of the constructed in [B1-B3] spectral decomposition of the set $\overline{\operatorname{Per} f}$ (slightly different versions of the decomposition for the union of all $\omega$-limit sets of $f$ and for the nonwandering set of $f$ may also be found in [B1-B3]). We state in this paper only the part of the decomposition theorem concerning $\overline{\operatorname{Per} f}$. Indeed, it was proven in [S] that $\overline{\operatorname{Per} f}$ is the center of an interval map $f$ (see also [N]; in fact for all maps $f \in \mathscr{P}$ it follows from Theorem 1). At the same time the definitions show that to study f-rotation numbers and sets it is sufficient to know the properties of the center of $f$ which justifies our approach.

Decomposition Theorem ([B1-B3]). Let $f \in \mathscr{P}$. Then there exist an at most countable family of basic sets $\left\{B_{i} \subset B_{i}^{\prime}\right\}$ and a family of triplets of solenoidal sets $\left\{S^{(\alpha)} \subset S_{p}^{(\alpha)} \subset Q^{(\alpha)}\right\}_{\alpha \in A}$ such that: 
(1) $\overline{\operatorname{Per} f}=X_{f} \cup\left(\bigcup_{\alpha} S_{p}^{(\alpha)}\right) \cup\left(\bigcup_{i} B_{i}\right)$ with intersections finite and possible only between different basic sets each three of which have the empty intersection;

(2) the set $\left\{\alpha\right.$ : int $\left.Q^{(\alpha)} \neq \varnothing\right\}$ is at most countable and $S^{(\alpha)}=Q^{(\alpha)}$ for all other $\alpha \in A$.

Finally we state the results which describe types of periodic orbits for zero entropy one-dimensional maps. First results of this sort are due to Misiurewicz and Szlenk and then Misiurewicz [MS, M1] who showed that interval maps with zero entropy are exactly those whose periodic points have periods which are powers of 2 . Then Block [B12] proved that periodic orbits of an interval zero entropy map are simple (Block's results were extended for tree maps in [B4]). Finally, similar results for the circle maps follow from [ALM, BCJM and MZ1]. We summarize all the facts we need about zero entropy circle and interval maps in Proposition 7.

Proposition 7 ([ALM, Bl2, BCJM, M1, MS, MZ1]). Let $f: X \rightarrow X, f \in$ $\mathscr{P}, M \subset X$ be a cycle of intervals or $M=X=S^{1}, h(f \mid M)=0$. Then there is a k-periodic pattern $\pi$ represented by an $f$-periodic orbit and $n \leq \infty$ such that any cycle $S$ of $f \mid M$ represents a simple extension of $\pi$ of period $2^{i} k, i<n$ and for any $i<n$ there is a cycle of $f \mid M$ of period $2^{i} k$. Moreover, if $M \neq S^{1}$ then $M$ represents $\pi$.

\section{Proofs of Main Results.}

We have already deduced Propositions 1-3 from Theorem 1, thus only Theorem 2 has to be proven. In case of a circle map with no periodic points Theorem 2 follows from Proposition 1 so we may assume that $f \in \mathscr{P}$. Before we begin let us outline the main idea which in fact works in some other one-dimensional situations (see, e.g., [B5]) and allows us to connect the properties of arbitrary one-dimensional maps with those of zero entropy maps. Suppose that $f$ is a one-dimensional map, consider all the cycles of intervals generating basic sets and order them by inclusion. Then the entropy of $f$ is zero on $\omega$-limit sets of points which never get into the above-mentioned cycles of intervals. At the same time one can change the map on maximal among these cycles of intervals so that they remain cycles of intervals for the new map and the new map on them has the zero entropy too. Thus the new map will have zero entropy and so the maximal cycles of intervals for the original map must represent patterns allowed for zero entropy maps.

More precisely, we show that one can choose periodic orbits in maximal cycles of intervals which represent patterns of these cycles of intervals and then change the map outside these periodic orbits so that the resulting map is of zero entropy. We apply these arguments in more detailed form in the proof of Proposition 9 but first let us prove the following

Proposition 8. Let $B=B(M, f)$ be a basic set of $f: K \rightarrow K, \phi: K \rightarrow \mathbb{R}^{n}$ be a function. Then the sets $V_{f \mid B}(\phi)$ and $I_{f \mid B}(\phi)$ are connected. Moreover, if $\phi$ satisfies the conditions of Theorem 2 then the following holds:

(1) if $P \subset B$ is a periodic orbit then $\rho_{f, \phi}(P) \in I_{f \mid B}(\phi)$;

(2) if $L \subset M$ is a complementary to $B(M, f)$ in $M$ cycle of intervals and $Q \subset L$ is a periodic orbit such that $\operatorname{per}(Q)=\operatorname{per}(L)$ then $\rho_{f, \phi}(Q) \in I_{f \mid B}(\phi)$. 
Proof. Let $\nu, \mu \in V_{f \mid B}(\phi)$. Then for any convex combination $\eta=a \nu+b \mu$ we have that $\eta\left(D_{\phi}\right)=0$. At the same time by Theorem 1 and Proposition 5 there is a point $x \in B$ generic for $\eta$. Thus $\eta \in V_{f \mid B}(\phi)$ and $V_{f \mid B}(\phi)$ is connected which implies that $I_{f \mid B}(\phi)$ is connected.

Suppose that $\phi$ satisfies the conditions from Theorem 2 and prove statement (1). Let $P \subset B$ be a periodic orbit. If $K$ is the circle then $\phi$ is continuous at the points of $P$, so $\rho_{f, \phi}(P) \in I_{f \mid B}(\phi)$ and statement (1) is proven. If $K=[0,1]$ there are two cases. If $P$ is not simple then $\phi$ is continuous at all the points of $P$ and $\rho_{f, \phi}(P) \in I_{f \mid B}(\phi)$. Let $P$ be simple. Then $M$ is also simple, $\operatorname{per}(M)=2^{n}$ for some $n$. The following arguments are an easy version of those we are going to employ later on. Namely, by Proposition 5 there is a transitive nonstrictly periodic map $f^{\prime}: M^{\prime} \rightarrow M^{\prime}$ such that $f \mid B$ is almost conjugate to $f^{\prime} \mid M^{\prime}$ by a map $\xi$. We would like to apply some well-known results for interval maps so let us extend $f^{\prime}$ to the map $g:[0,1] \rightarrow[0,1]$ with $g\left|M^{\prime}=f^{\prime}\right| M^{\prime}$ such that $g$ is linear on each component of $[0,1] \backslash M^{\prime}$; note however that this step is not essential and needed only to enable us to formally apply the aforementioned results established for interval maps but not for nonstrictly peridoic maps. The properties of simple patterns imply that outside $M^{\prime}$ the map $g$ has periodic points of periods $2^{i}, i<n$, and only them. Also for obvious reasons $g \mid M^{\prime}$ has a point of period $2^{n}$ and by Proposition 6 $g \mid M^{\prime}$ has points of all periods $2^{n+1} m, m>0$, so $g$ has periodic orbits of all periods $2^{t}, t>0$.

It is known [MN] that then $g$ has a simple periodic orbit of period $2^{t}$ for any $t$. Pick up such $t>n$ that $t, t+1 \in A$ (see the properties of the function $\phi$ ); let $P^{\prime}$ be a simple $g$-periodic orbit of period $2^{t}$. Then $P^{\prime} \in M^{\prime}$ and we can consider the $\xi$-preimage of $P^{\prime}$ which is a complementary to $B$ in $M$ cycle of intervals of the same pattern. So by Corollary 6 there is a simple periodic orbit $P^{\prime} \subset B$ of period $2^{t}$ or $2^{t+1}$. In any case $\phi$ is continuous at all the points of $P^{\prime \prime}$ and so $\rho_{f, \phi}\left(P^{\prime \prime}\right) \in I_{f \mid B}(\phi)$. Clearly $P$ and $P^{\prime \prime}$ are equivalent, so by the properties of $\phi$ we have $\rho_{f \mid B, \phi}\left(P^{\prime \prime}\right)=\rho_{f \mid B, \phi}(P)$ and thus we get the required. Statement (2) now follows from statement (1) and Corollary 6.

Proposition 9. Let $f: K \rightarrow K$ be a nonstrictly periodic or a circle map and $\mathscr{N}_{f}=\mathscr{N}$ be the family of all generating basic sets cycles of intervals $N \subset K$ such that if $B\left(K^{\prime}, f\right)$ is a basic set and $K^{\prime} \supset N$ then $K^{\prime}$ is a cycle of intervals and $\operatorname{per}\left(K^{\prime}\right)=\operatorname{per}(N)$. Furthermore, let $\mathscr{N}_{f}^{\prime}=\mathscr{N}^{\prime}$ be the set of periodic orbits which consist of endpoints of cycles of intervals from $\mathscr{N}$ and $\mathscr{D}_{f}=\mathscr{D}$ be the family of all periodic orbits which do not belong to any set $M$ generating a basic set. Then there is a t-periodic pattern $\gamma(f)=\gamma$ represented by an $f$-periodic orbit and an $n \leq \infty$ such that any element of $\mathscr{N} \cup \mathscr{N}^{\prime} \cup \mathscr{D}$ represents a simple extension of $\gamma$ of period $t 2^{i}, i<n$, and if $i<n$ then there is an element of $\mathscr{N} \cup \mathscr{N}^{\prime} \cup D$ which represents a simple extension of $\gamma$ of period $t 2^{i}$; if $K$ is a cycle of intervals then $\gamma=\operatorname{ptn}(K)$ and if $K$ is the circle then $\gamma$ depends on $f$.

Proof. Let $N \in \mathscr{N}, \operatorname{per}(N)=n$. Consider a family $\mathscr{S}_{N}$ of all cycles of intervals which generate basic sets and contain $N$. Obviously $\mathscr{S}_{N} \subset \mathcal{N}$; moreover, $\operatorname{per}(S)=n$ and $\operatorname{ptn}(S)=\operatorname{ptn}(N)$ for any $S \in \mathscr{S}_{N}$. Let $A_{N}=\bigcup_{S \in \mathscr{S}_{N}} S$ and show that if $S^{\prime} \in \mathcal{N}$ and $S^{\prime} \subset A_{N}$ then $\operatorname{ptn}\left(S^{\prime}\right)=\operatorname{ptn}(N)$. Indeed, by Proposition $5 B\left(S^{\prime}, f\right)$ is noncountable and by Decomposition Theorem the family of 
all basic sets is countable, thus there is $M \in \mathscr{S}_{N}$ such that $B\left(S^{\prime}, f\right) \cap$ int $M \neq \varnothing$ and so $S^{\prime} \subset M$; this implies that $\operatorname{per}\left(S^{\prime}\right)=n$ and therefore the required. Assume that there exists a cycle of intervals $N \in \mathscr{N}$ such that $A_{N}=K$. Then by what we just showed $\operatorname{ptn}\left(S^{\prime \prime}\right)=\operatorname{ptn}(N)$ for any $S^{\prime \prime} \in \mathcal{N}$. Moreover if $K$ is a cycle of intervals then there is a periodic orbit $Q$ which represents $\operatorname{ptn}(K)$. At the same time since $K=A_{N}$ there is $S^{\prime \prime} \in \mathscr{N}$ which contains $Q$ and so $\operatorname{ptn}\left(S^{\prime \prime}\right)=\operatorname{ptn}(K)=\operatorname{ptn}(N)=\gamma(f)$. Clearly $\mathscr{D}_{f}$ is empty in this case and also a cycle from $\mathscr{N}^{\prime}$ (if any) represents either $\operatorname{ptn}(K)$ or a simple exntension of $\operatorname{ptn}(K)$. This finishes the consideration of the case $A_{N}=K$.

From now on we assume that $A_{N} \neq K$ for any $N \in \mathscr{N}$ which implies that $A_{N}$ is a cycle of intervals (not necessarily closed). Let us show that $\operatorname{per}\left(A_{N}\right)=$ $n$. Indeed, if $\operatorname{per}\left(A_{N}\right)=m<n$ and $A^{\prime}$ is one of components of $A$ then $f^{m} A^{\prime}=A^{\prime}$. Since $A^{\prime}$ contains infinitely many points from basic sets of $f$ there is an $f^{m}$-fixed point $x \in$ int $A^{\prime}$; clearly $x \in S$ for some cycle of intervals $S \in \mathscr{S}_{N}$ which contradicts the fact that $\operatorname{per}(S)=n$. Thus $\operatorname{per}\left(A_{N}\right)=n$. Note that $\bigcup_{N \in \mathcal{N}} A_{N}$ contains all cycles of intervals generating basic sets.

Consider a process of changing $f$ which leads to a zero entropy map $g$ such that for any $N \in \mathscr{N}$ the map $g$ has at least one periodic point of pattern $\operatorname{ptn}(N)$. Let us fix $N_{1} \in \mathscr{N}$ and change $f$ only on $A_{N_{1}}=A_{1}$ so that the new map $f_{1}$ is such that $f_{1}\left|Q\left(N_{1}\right)=f\right| Q\left(N_{1}\right), f_{1}\left|\partial A_{1}=f\right| \partial A_{1}$ and $f_{1} \mid A_{1}$ is linear on every component of $A_{1} \backslash Q\left(N_{1}\right)$ (the definition of a periodic orbit $Q(M)$ which exists for any cycle of intervals $M$ generating basic sets was given earlier; its main properties are described in Corollary 7). Clearly $f_{1}$ is continuous: Consider an $f$-cycle of intervals $N_{2} \in \mathscr{N}$ such that $N_{2} \not \subset A_{1}$ (if any); let us show that $Q\left(N_{2}\right)$ is disjoint from $A_{1}$. Indeed, $A_{1} \not \subset N_{2}$ since otherwise the definition implies that $N_{2} \in \mathscr{S}_{N_{1}}$ and so $N_{2} \subset A_{1}$. Now if $Q\left(N_{2}\right) \subset A_{1}$ then the mutual disposition of $N_{2}$ and $A_{1}$ implies by Corollary 7 that int $A_{1}$ contains points from $B\left(N_{2}, f\right)$ and so $N_{2} \subset A_{1}$ which is a contradiction, so indeed $Q\left(N_{2}\right)$ and $A_{1}$ are disjoint.

Let us consider the set $A_{2}=A_{N_{2}}$ and change $f_{1}$ as follows.

(1) We change the map only on $A_{2} \backslash A_{1}$ leaving it as it is on $Q\left(N_{2}\right)$ and at all the endpoints of components of $A_{2}$ which do not belong to $A_{1}$.

(2) The new map $f_{2}$ is linear on every component of $A_{2} \backslash\left(A_{1} \cup Q\left(N_{2}\right)\right)$.

Clearly the construction is possible and $f_{2}$ is continuous. Moreover, the only patterns represented by $f_{2}$-periodic orbits in $A_{1} \cup A_{2}$ are those of $N_{1}, N_{2}$ and, perhaps, their 2-extensions, so there are finitely many of them, and it is easy to see that in fact any point from $A_{1} \cup A_{2}$ converges to one of the aforementioned periodic orbits. Finally the construction implies that for any component of $A_{1} \cup A_{2}$ its $f_{2}$-image belongs to its $f$-image (for by the construction the former is in fact a convex hull of $f$-images of some points from the component).

Going on with this construction we find on the step $r$ an $f$-cycle of intervals $N_{r} \in \mathscr{N}$ such that $N_{r} \not \subset \bigcup_{i=1}^{r-1} A_{i}$ (if such $N_{r}$ does not exist the construction is finished). Then the same reasons as before show that we can continue; since there are at most countably many basic sets we can organize the process so that for any cycle of intervals $M \in \mathscr{N}$ there is a number $r$ such that $M \subset A_{r}$. On every step in the construction the following properties will be held. First, all the patterns of periodic orbits which appear in the construction are either patterns represented by cycles of intervals $M \in \mathscr{N}$ or their 2-extensions (the latter may 
happen because we keep the map unchanged at the appropriate endpoints of sets $A_{M}$ and sometimes all these endpoints form a periodic orbit which represents a 2-extension of $\operatorname{ptn}(M))$. Second, all the points from $\bigcup_{i=1}^{n} A_{i}$ converge to periodic orbits. Third, by the same reasons as before for any component of $\bigcup_{i=1}^{n} A_{i}$ its $f_{n}$-image belongs to its $f$-image. Finally, by the construction the map $f_{n+1}$ is obtained by changing $f_{n}$ on the set $A_{n+1} \backslash A_{n}$; obviously we can choose sets $N_{r}$ (and so $A_{r}$ ) in such an order that the length of the longest component of $A_{n+1} \backslash A_{n}$ will converge to 0 as $n$ approaches infinity.

Let $\bigcup_{i \geq 1}$ int $A_{i}=W$ and int $\left(K \backslash \bigcup_{i \leq 1} A_{i}\right)=V$. Let us define a map $g$ and then show that $f_{r}$ uniformly converges to $g$ and so $g$ is continuous. By the construction all $f_{n}$ coincide with $f$ outside $W$; let $g=f$ outside $W$. Also, for any point $x \in W$ there is the smallest $r$ such that $x \in$ int $A_{r}$. Then by the construction $f_{r}(x)=f_{m}(x)$ for all $m \geq r$; let $g(x)=f_{r}(x)$. Clearly $g$ is now defined everywhere. Let us show that $f_{r}$ uniformly converges to $g$ as $r$ tends to infinity. Indeed, by the construction $f_{n}$ may not coincide with $g$ only at points from the union of the sets $\bigcup_{r=n}^{\infty} A_{r+1} \backslash A_{r}$. If $n$ is big and $r \geq n$ then any component $K$ of any set $A_{r+1} \backslash A_{r}$ is short, and so the $f$-image of $K$ is short too which by the construction implies that $f_{r+1}$-image of $K$ is also short. The way we define $g$ implies now that $g-f_{n}$ is small everywhere, and so indeed $f_{n}$ converges to $g$ as $n$ tends to infinity, which implies that $g$ is continuous.

Clearly $h(g)=0$. Indeed, by the construction if $g^{n} x \in W$ for some point $x$ and some $n$ then the set $\omega_{g}(x)$ is a $g$-periodic orbit, so $h(g \mid \omega(x)=$ 0 . Otherwise $g$ coincides with $f$ on the orbit of $x$ which never enters $W$; thus $\omega_{g}(x)=\omega_{f}(x)=H$ is disjoint from $W, g|H=f| H$ and so if $h(g \mid H) \neq 0$ then $h(f \mid H) \neq 0$ and by Decomposition Theorem there is a basic set $B\left(M^{\prime \prime}, f\right) \sup H$. If the orbit of $x$ enters int $M^{\prime \prime}$ then by the construction it enters $W$ which contradicts the assumption. On the other hand if $x$ does not enter int $M^{\prime \prime}$ then $B\left(M^{\prime \prime}, f\right) \sup H$ implies that $\omega(x)=H$ is a periodic orbit which contradicts $h(g \mid H)=h(f \mid H)>0$. Now, by the construction the family of all patterns represented by $g$-periodic orbits coincides with the family of all patterns represented by cycles of intervals $N \in \mathscr{N}$ (and so by the periodic orbits $Q(N)$ ), by some $f$-periodic orbits which consist of endpoints of cycles of intervals $N \in \mathscr{N}$ or by periodic orbits from $\mathscr{D}$. Since an $f$-periodic orbit which consists of endpoints of a cycle of intervals $N \in \mathscr{N}$ represents either the same pattern as $N$ or its 2-extension then by Proposition 7 we get the required.

Proposition 9 covers the family of nonstrictly periodic maps or circle maps $f$ such that the basic set $B\left(S^{1}, f\right)$ does not exist; indeed, if $f: S^{1} \rightarrow S^{1}$ and $B\left(S^{1}, f\right)$ exists then the families $\mathscr{N}_{f}, \mathcal{N}_{f}^{\prime}$ and $\mathscr{D}_{f}$ are empty. Proposition 9 implies Corollary 8 which may be considered as an extension of Proposition 7 (the pattern $\gamma(f)$ in Corollary 8 replaces the pattern $\pi$ from Proposition 7 ).

Corollary 8. Let $f: K \rightarrow K$ be a nonstrictly periodic or a circle map, $\mathscr{F}$ be the family of periodic orbits such that if $P \in \mathscr{F}$ and $M \supset P$ is a set which generates a basic set then $M$ is a cycle of intervals of period $\operatorname{per}(M)=\operatorname{per}(P)$. Then there is a pattern $\gamma$ such that any $P \in \mathscr{F}$ represents a simple extension of $\gamma$, and if $f$ is nonstrictly periodic then $\gamma=\operatorname{ptn}(K)$. 
The next proposition seems to be instrumental in the proof of Theorem 2; it also adds information to Decomposition Theorem. Before we state it let us introduce the following definition: a pattern $\pi$ is an almost simple extension of a pattern $\pi^{\prime}$ if either $\pi$ is a simple extension of $\pi^{\prime}$ or there is a pattern $\theta$ such that $\pi$ is a simple extension of $\theta$ and $\pi^{\prime}$ is a 2-extension of $\theta$.

Proposition 10. Let $f: K \rightarrow K$ be a nonstrictly periodic or a circle map, $Q$ be a periodic orbit. Then there is a finite nested sequence of sets $Q=N_{0} \subset N_{1} \subset$ $\cdots \subset N_{k}$ such that the following holds:

(1) $N_{i}$ are cycles of intervals and $\operatorname{per}\left(N_{i}\right)>\operatorname{per}\left(N_{i+1}\right)$ for $0 \leq i<k$ except for the case when $i=k-1, N_{i+1}=N_{k}$ is the circle and so $\operatorname{per}\left(N_{k}\right)$ is not defined;

(2) $N_{i}$ generates a basic set for $0<i \leq k$;

(3) for any $i<k$ there is a periodic orbit $P_{i+1} \subset B\left(N_{i+1}, f\right)$ such that $\operatorname{ptn}\left(N_{i}\right)$ is an almost simple extension of $\operatorname{ptn}\left(P_{i+1}\right)$;

(4) if $f$ is a nonstrictly periodic map or a circle map with no basic set $B\left(S^{1}, f\right)$ then $N_{k}$ is a cycle of intervals and $\operatorname{ptn}\left(N_{k}\right)$ is a simple extension of $\gamma(f)$ while if $f$ is a circle map and $B\left(S^{1}, f\right)$ exists then $N_{k}=S^{1}$.

Proof. Let $\mathscr{M}(Q)=\mathscr{M}_{1}$ be a family of cycles of intervals $M$ generating basic sets $B(M, f)$ such that $Q \subset M$ and $\operatorname{per}(Q)>\operatorname{per}(M)$. Suppose that $\mathscr{M}_{1}=\varnothing$ and consider some cases. If $f: S^{1} \rightarrow S^{1}$ is a circle map and the basic set $B\left(S^{1}, f\right)$ exists then there are two possibilities. First, $Q \subset B\left(S^{1}, f\right)$; then set $k=1$ and $N_{1}=S^{1}$. Second, $Q$ is disjoint from $B\left(S^{1}, f\right)$. Then by Proposition 5 there is a cycle of intervals $L \supset Q$ complementary to $B\left(S^{1}, f\right)$; furthermore, the assumption that $\mathscr{M}_{1}=\varnothing$ implies that for a cycle of intervals $M$ generating basic sets $B(M, f)$ such that $Q \subset M$ (if any) we have $\operatorname{per}(M)=$ $\operatorname{per}(L)$. So by Corollary 8 applied to $f \mid L$ we see that $\operatorname{ptn}(Q)$ is a simple extension of $\operatorname{ptn}(L)$ and by Corollary 6 there is a periodic orbit $P_{1} \subset B\left(S^{1}, f\right) \cap$ $L$ such that $Q$ is an almost simple extension of $P_{1}$. Clearly setting $N_{1}=S^{1}$ we get the required. Let now $f$ be either a circle map without the basic set $B\left(S^{1}, f\right)$ or a nonstrictly periodic map; since $\mathscr{M}_{1}$ is empty then Corollary 8 is applicable and so $\operatorname{ptn}(Q)$ is a simple extension of $\gamma(f)$. Setting $k=0$ and $Q=N_{0}$ we finish consideration of this case.

Suppose that $\mathscr{M}_{1} \neq \varnothing$; take a cycle of intervals $N_{1} \in \mathscr{M}_{1}$ of the biggest period $p_{1}$ and show that there is a periodic orbit $P_{1} \in B\left(N_{1}, f\right)$ such that $\operatorname{ptn}(Q)$ is an almost simple extension of $\operatorname{ptn}\left(P_{1}\right)$. We may assume that $Q$ is disjoint from $B\left(N_{1}, f\right)$. Then by Proposition 5 there is a cycle of intervals $L \supset Q$ complementary to $B\left(N_{1}, f\right)$ in $N_{\mathrm{i}}$. Let us show that $\operatorname{ptn}(Q)$ is a simple extension of $\operatorname{ptn}(L)$. Indeed, if $L^{\prime \prime \prime}$ is a cycle of sets generating a basic set and such that $Q \subset L^{\prime \prime \prime} \subset L$ then by the choice of $p_{1}$ and $N_{1}$ we have $\operatorname{per}\left(L^{\prime \prime \prime}\right)=$ $\operatorname{per}(Q)$. Thus by Corollary $8 \operatorname{ptn}(Q)$ is a simple extension of $\operatorname{ptn}(L)$. At the same time by Corollary 6 there is a periodic orbit $P_{1} \in B\left(N_{1}, f\right) \cap L$ such that either $\operatorname{ptn}\left(P_{1}\right)=\operatorname{ptn}(L)$ or $\operatorname{ptn}\left(P_{1}\right)$ is a 2-extension of $\operatorname{ptn}(L)$, so we see that $\operatorname{ptn}(Q)$ is an almost simple extension of $\operatorname{ptn}\left(P_{1}\right)$. Note that by the construction $\operatorname{per}(Q)>\operatorname{per}\left(N_{1}\right)=p_{1}$.

Now we can repeat the same arguments for the periodic orbit $Q\left(N_{1}\right)$ replacing $Q$. Note that the cycle of intervals $N_{2}$ which we find will have period smaller that $\operatorname{per}\left(Q\left(N_{1}\right)\right)=\operatorname{per}\left(N_{1}\right)$ and will contain $Q\left(N_{1}\right)$, so by Corollary 7 $N_{1} \subset N_{2}$. The strict decreasing of periods of cycles of intervals $N_{i}$ implies that 
for some $j$ the family $\mathscr{M}\left(Q\left(N_{j}\right)\right)$ of cycles of intervals $M$ generating basic sets $B(M, f)$ such that $Q\left(N_{j}\right) \subset M$ and $\operatorname{per}(M)<\operatorname{per}\left(Q\left(N_{j}\right)\right)$ will be empty. Due to what we saw in the first paragraph of the proof if $f: S^{1} \rightarrow S^{1}$ is a circle map and the basic set $B\left(S^{1}, f\right)$ exists then we can set $k=j+1, N_{k}=S^{1}$ and get the required; otherwise as we saw $\operatorname{ptn}\left(Q\left(N_{j}\right)\right)$ is a simple extension of $\gamma(f)$, so we can set $j=k$ and again get the required which concludes the proof.

\section{We are ready now to prove Theorem 2 .}

Proof of Theorem 2. If $f$ is a circle map without periodic points then Theorem 2 follows from Theorem AK so we may assume $f$ has periodic points. If $f$ has finitely many periods then $h(f)=0$ (see, for example, [Bl1] or [ALM]); it also follows from the fact that in this case there are no basic sets. Now by Proposition 7 periodic points of $f$ represent extensions of $\gamma(f)$ (note that in the interval case $\gamma(f)$ is a fixed-point pattern). Then Theorem 2 follows from the definitions, so we may assume that the set of periods of periodic points of $f$ is infinite. Clearly, there are two cases: (1) $f: S^{1} \rightarrow S^{1}$ is a circle map and there exists a basic set $B\left(S^{1}, f\right) ;(2) f$ is either a circle map with no basic set $B\left(S^{1}, f\right)$ or an interval map.

Our first step in the proof is to construct a special set $C$. In case (1) let $C=I_{f \mid B\left(S^{1}, f\right)}(\phi)$; then $C \subset I_{f}(\phi)$ and by Proposition $8 C$ is connected. Consider case (2). Assume that the period of $\gamma(f)$ is $k$ and show that there are periodic orbits of all periods $2^{i} k, k \geq 0$, which represent simple extensions of $\gamma(f)$. Indeed, the set of periods of periodic points of $f$ is infinite. If at the same time the entropy $h(f)=0$ then we obtain the required by Proposition 7 . Let $h(f)>0$. Then by Proposition 9 there is an $n \leq \infty$ and periodic points of $f$ of all periods $2^{i} k, i<n$, which represent simple extensions of $\gamma(f)$. Clearly, we may assume that $n<\infty$. The fact that $h(f)>0$ implies then that for some $i<n$ there is a cycle of intervals of period $2^{i} k$ which represents a simple extension of $\gamma(f)$ and generates a basic set $B(M, f)$. By Proposition $6 f \mid M$ has periodic orbits of all periods $2^{j} k, j \geq i$; now the description of primary cycles for interval and circle maps [BCMJ, ALM] implies that in fact $f \mid M$ has periodic orbits of all periods $2^{j} k, j \geq i$, which are simple extensions of $\gamma(f)$. This shows that if the set of periods of periodic points of $f$ is infinite then $f$ has periodic orbits of all periods $2^{r} k, 0 \leq r<\infty$, which are simple extensions of $\gamma(f)$. Then there is a periodic orbit $P$ such that $\operatorname{ptn}(P)=\gamma(f)$; let $C=\left\{\rho_{f, \phi}(P)\right\}=\{c\}$. The properties of $\phi$ imply that $\rho_{f, \phi}\left(P^{\prime}\right)=c$ for any periodic orbit $P^{\prime}$ such that $\operatorname{ptn}\left(P^{\prime}\right)$ is a simple extension of $\gamma(f)$ and also that there is at least one such periodic orbit on which $\phi$ is continuous. So we conclude that in this case also $C \subset I_{f}(\phi)$.

We show that for a periodic orbit $Q$ there is a connected set $G$ nondisjoint from $C$ such that $\rho=\rho_{f, \phi}(Q) \in G \subset I_{f}(\phi)$; the density of rotation numbers of periodic points in $I_{f}(\phi)$ then implies that $I_{f}(\phi)$ is connected. Let $Q=N_{0} \subset N_{1} \subset \cdots \subset N_{k}$ be the sequence of sets from Proposition $10, I_{j}=I_{f \mid B\left(N_{j}, f\right)}(\phi)$ and show that $\rho_{j+1}^{\prime}=\rho_{f, \phi}\left(P_{j+1}\right) \in I_{j} \cap I_{j+1}$ for any $j<k$. Indeed, by Corollary 5 there is a periodic orbit $P_{j}^{\prime} \subset B\left(N_{j}, f\right)$ such that either $\operatorname{ptn}\left(P^{\prime}\right)_{j}=\operatorname{ptn}\left(Q\left(N_{j}\right)\right)=\operatorname{ptn}\left(N_{j}\right)$ or $\operatorname{ptn}\left(P_{j}^{\prime}\right)$ is a 2 extension of $\operatorname{ptn}\left(Q\left(N_{j}\right)\right)=\operatorname{ptn}\left(N_{j}\right)$. In either case by Proposition $10 \operatorname{ptn}\left(P_{j}^{\prime}\right)$ is an almost 
simple extension of $\operatorname{ptn}\left(P_{j+1}\right)$, so $\rho_{f, \phi}\left(P_{j}^{\prime}\right)=\rho_{j+1}^{\prime}$. By Proposition 8 applied to both $P_{j}^{\prime}$ and $P_{j+1}$ we now have that $\rho_{j+1}^{\prime} \in I_{j} \cap I_{j+1}$. Thus the set $G=\bigcup_{j=1}^{k} I_{j}$ is connected since each $I_{j}$ is connected by Proposition 8 . At the same time by Proposition 10 and the definition of $C$ we see that $I_{k} \cap C \neq \varnothing$ which concludes the proof.

\section{REFERENCES}

[ALM] L. Alseda, J. Llibre, and M. Misiurewicz, Combinatorial dynamics and entropy in dimension one, World Scientific, Singapore, 1993.

[AK] J. Auslander and Y. Katznelson, Continuous maps of the circle without periodic points, Israel J. Math. 32 (1979), 375-381.

[B11] L. Block, Continuous maps of the Interval with finite nonwandering set, Trans. Amer. Math. Soc. 240 (1978), 221-230.

[B12] - Simple periodic orbits of mappings of the interval, Trans. Amer. Math. Soc. 254 (1979), 391-398.

[BCJM] L. Block, E. Coven, L. Jonker, and M. Misiurewicz, Primary cycles on the circle, Trans. Amer. Math. Soc. 311 (1989), 323-335.

[B1] A. M. Blokh, Decomposition of dynamical systems on an interval, Russian Math. Surveys 38 (1983), no. 5, 133-134.

[B2] _ On dynamical systems on one-dimensional branched manifolds. I, II, III, Teor. Funktsii Funktsional. Anal. i Prilozhen No. 46 (1986), 8-18; No. 47 (1986), 67-77; No. 48 (1987), 32-46. (Russian)

[B3] _ The spectral decomposition for one-dimensional maps, SUNY at Stony Brook, Preprint \#1991/14, September, Dynamics Reported (to appear).

[B4] _ Trees with snowflakes and zero entropy maps, Topology 33 (1994), 379-396.

[B5] _ On some properties of graph maps: pectral decomposition, Misiurewicz conjecture and abstract sets of periods, Max-Planck-Institut für Mathematik, Preprint \#35, June 1991.

[B6] _ Rotation numbers, twists and a Sharkovskil-Misiurewicz-type ordering for patterns on the interval, Ergodic Theory Dynamical Systems (to appear).

[DGS] M. Denker, C. Grillenberger, and K. Sigmund, Ergodic theory on compact spaces, Lecture Notes in Math., vol. 527 , Springer, Berlin, 1976.

[I] R. Ito, Rotation sets are closed, Math. Proc. Cambridge Philos. Soc. 89 (1981), 107-111.

[M1] M. Misiurewicz, Horseshoes for mappings of the interval, Bull. Acad. Polon. Sci. Ser. Sci. Math. 27 (1979), 167-169.

[M2] _ Periodic points of maps of degree one of a circle, Ergodic Theory Dynamical Systems 2 (1982), 221-227.

[MS] M. Misiurewicz and W. Szlenk, Entropy of piecewise monotone mappings, Studia Math. 6 (1980), 45-53.

[MN] M. Misiurewicz and Z. Nitecki, Combinatorial patterns for maps of the interval, Mem. Amer. Math. Soc. 456 (1990).

[MZ1] M. Misiurewicz and K. Ziemian, Cycles for degree -1 circle maps, European Conference on Iteration Theory (ECIT 87), World Scientific, Singapore, 1989, pp. 8-25.

[MZ2] _ _ Rotation sets for maps of tori, J. London Math. Soc. (2) (1989), 490-506.

[N] Z. Nitecki, Periodic and limit orbits and the depth of the center for piecewise monotone interval maps, Proc. Amer. Math. Soc. 80 (1980), 511-514.

[NPT] S. Newhouse, J. Palis, and F. Takens, Bifurcations and stability of families of diffemorphisms, Inst. Hautes Études Sci. Publ. Math. 57 (1983), 5-71.

[P] H. Poincaré, Sur les courbes definies par les equations differentielle, Oeuvres Completes, vol. 1, Gauthier-Villars, Paris, 1952, pp. 137-158. 
[S] A. N. Sharkovskii, Non-wandering points and the center of a continuous map of the line into itself, Dopovīdī Akad. Nauk Ukraïn. RSR Ser. A (1964), 865-868. (Ukrainian)

[Z] K. Ziemian, in preparation.

Department of Mathematics, University of Alabama at Birmingham, UAB Station, Birmingham, Alabama 35294-2060

E-mail address: ablokh@math.uab.edu 